\title{
Dominance of the Enterocytozoon bieneusi genotype BEB6 in red deer (Cervus elaphus) and Siberian roe deer (Capreolus pygargus) in China and a brief literature review
}

\author{
Wei Zhao, Jianguang Wang, Ziyin Yang, and Aiqin Liu* \\ Department of Parasitology, Harbin Medical University, Harbin, Heilongjiang 150081, PR China
}

Received 9 August 2017, Accepted 3 December 2017, Published online 21 December 2017

\begin{abstract}
Enterocytozoon bieneusi is the most frequently diagnosed microsporidian species in humans and is also found in a wide range of animals. It is considered to be an important but neglected zoonotic pathogen. With the development of deer bred in captivity, the number of deer has been increasing in recent years in China and there are more people involved in this work. The aims of this study were to determine prevalence and genotypes of E. bieneusi in red deer (Cervus elaphus) and Siberian roe deer (Capreolus pygargus), and to assess their potential zoonotic transmission. A total of 122 fecal specimens were collected from 104 red deer and 18 roe deer from three deer farms in Heilongjiang and Jilin Provinces, China. Enterocytozoon bieneusi was detected and genotyped by PCR and sequencing of the internal transcribed spacer (ITS) region of the rRNA gene. The average infection rate was $8.2 \%(10 / 122)$, with $7.7 \%(8 / 104)$ for red deer and $11.1 \%(2 / 18)$ for roe deer. Two genotypes were identified: a known genotype BEB6 $(n=9)$ and a novel genotype named HLJD-VI $(\mathrm{n}=1)$. This is the first report of E. bieneusi infection in Siberian roe deer. The fact that genotype BEB6 was detected previously in one human case of microsporidiosis, and that genotype HLJD-VI fell into zoonotic group 1, suggest the possibility of transmission to humans. A brief review of E. bieneusi genotypes in deer worldwide shows that 40 genotypes have been found in seven deer species, with genotype BEB6 being predominant.
\end{abstract}

Keywords: Enterocytozoon bieneusi, Genotyping, ITS region, Red deer, Siberian roe deer, Zoonotic

Résumé - Dominance du génotype BEB6 d'Enterocytozoon bieneusi chez le cerf élaphe (Cervus elaphus) et le chevreuil de Sibérie (Capreolus pygargus) en Chine et une brève revue de la littérature. Enterocytozoon bieneusi est l'espèce de microsporidie la plus fréquemment diagnostiquée chez l'homme et se retrouve également chez un large éventail d'animaux. Elle est considérée comme un pathogène zoonotique important mais négligé. Avec le développement de l'élevage de cerfs en captivité, le nombre de cerfs a augmenté ces dernières années en Chine et il y a plus de gens impliqués dans ce travail. Les objectifs de cette étude étaient de déterminer la prévalence et les génotypes d'E. bieneusi chez le cerf élaphe (Cervus elaphus) et le chevreuil de Sibérie (Capreolus pygargus) et d'évaluer leur potentiel de transmission zoonotique. Au total, 122 spécimens de fèces ont été prélevés de 104 cerfs et 18 chevreuils de trois élevages de cerfs dans les provinces du Heilongjiang et du Jilin, en Chine. Enterocytozoon bieneusi a été détecté et génotypé par PCR et séquençage de la région de l'ITS du gène de l'ARNr. Le taux moyen d'infection était de $8,2 \%(10 / 122)$, avec $7,7 \%(8 / 104)$ pour le cerf et 11,1\% (2/18) pour le chevreuil. Deux génotypes ont été identifiés: un génotype connu, BEB6 $(\mathrm{n}=9)$ et un nouveau génotype appelé HLJD-VI $(\mathrm{n}=1)$. Ceci est le premier rapport d'infection par E. bieneusi chez le chevreuil sibérien. Le fait que le génotype BEB6 ait été détecté précédemment dans un cas humain de microsporidiose et que le génotype HLJD-VI appartiennent au groupe zoonotique 1 suggère la possibilité d'une transmission à l'homme. Une brève revue des génotypes d'E. bieneusi chez les cerfs dans le monde montre que 40 génotypes ont été trouvés chez sept espèces de cerfs, le génotype BEB6 étant prédominant.

\footnotetext{
*Corresponding author: liuaiqin1128@126.com
} 


\section{Introduction}

The microsporidia, emerging as an important group of opportunistic pathogens, have a global distribution and at least 14 species are recognized as human pathogens [3]. Over $90 \%$ of human cases of microsporidiosis are caused by Enterocytozoon bieneusi [3]. E. bieneusi is one of the most significant causes of diarrheal diseases, especially in immunocompromized individuals [20]. E. bieneusi infections have been observed in many species of animals (mammals, birds and reptiles) [15,25]. Spores as the infective stage have been found in some environmental samples (water, soil and food) $[2,16]$. The epidemiology of human microsporidiosis caused by E. bieneusi is complex, and involves direct transmission (person-to-person and animal-to-person) and indirect transmission routes (through water, food and fomites contaminated with spores) [6].

Although light microscopy is commonly used in the diagnosis of microsporidia infections for stained clinical smears, especially for fecal samples, PCR-based molecular techniques have the ability to achieve an accurate diagnosis at species and genotype levels [9]. Currently, based on the high degree of diversity in the internal transcribed spacer (ITS) region of the ribosomalRNA (rRNA) gene within E. bieneusi, a standard method for genotyping E. bieneusi isolates has been established by sequence analysis of $243 \mathrm{bp}$ of the ITS region [24]. To date, more than 240ITS genotypes of E. bieneusi have been identified, while 33 of them are found in both humans and animals $[20,25,30]$. By phylogenetic analysis of nucleotide sequences of the ITS region, all the E. bieneusi genotypes can be unambiguously placed into nine distinct clades [12]. A large group (group 1) is composed of genotypes from both humans and animals, showing the zoonotic nature; the other eight groups (groups 2 to 9 ) mainly include genotypes from specific hosts or wastewater, showing host adaption to some extent [10]. To improve our understanding of the epidemiology of E. bieneusi and its populations, molecular epidemiological surveys should focus on genotyping E.bieneusi isolates from undersampled animal hosts.

Deer are one of the more common wild ruminant mammals. The number of deer bred in captivity has been increasing due to their economic significance to humans, and there are more people involved in this work. It has been reported that these animals play an important role in the epidemiology of E. bieneusi in some countries or areas [6]. Red deer (Cervus elaphus) are one of the most common deer species worldwide, while Siberian roe deer (Capreolus pygargus) are found within the temperate zone of eastern Europe and Asia. The two deer species are commonly found in China, especially in the northeastern part. Currently, there are no reports available on E. bieneusi infection in these animals. The present study was performed to determine the prevalence, genetic diversity, and zoonotic potential of E.bieneusi in captive red deer and roe deer in northeastern China.

\section{Material and Methods \\ Collection of fecal specimens}

During the period from November 2015 to March 2016, 122 fecal samples were collected including 44 red deer from Great Hinggan Mountains in Heilongjiang Province, and 60 red deer and 18 Siberian roe deer from Liaoyang City in Liaoning Province. The present study protocol was reviewed and approved by the Research Ethics Committee and the Animal Ethical Committee of Harbin Medical University. The inclusion of the farm animals in the present study was authorized by the farm manager. One fresh fecal specimen (approximately $30 \mathrm{~g}$ ) from each animal was collected from the ground immediately after defecation by sampling using a sterile disposal latex glove and then placing the sample into an individual plastic bag. Each individual was identified according to the ear tag, neck rope and body characteristics. All the fecal specimens were transported to the laboratory in a cooler with ice packs within 24 hours and stored in refrigerators at $4{ }^{\circ} \mathrm{C}$ prior to being used in molecular biological characterizations. Meanwhile, the age, gender and health status of each animal was recorded accordingly on the plastic bag. None of the animals showed clinical signs of illness at the time of sampling.

\section{DNA extraction and PCR amplification}

After the fecal specimens were sieved, the filtrates were concentrated and washed three times with distilled water by centrifugation for 10 minutes at $1500 \mathrm{~g}$. Genomic DNA was extracted from 180-200 mg washed fecal specimens using a QIAamp DNA Stool Mini Kit (QIAgen, Hilden, Germany) according to manufacturer-recommended procedures. DNA was eluted in $200 \mu \mathrm{L}$ of AE buffer and stored at $-20^{\circ} \mathrm{C}$ prior to PCR analysis.

Each of the DNA specimens was analyzed for the presence of E. bieneusi by nested PCR amplifying a $390 \mathrm{bp}$ nucleotide fragment of the rRNA gene including the $243 \mathrm{bp}$ ITS region, and the primer sequences and the cycling parameters in nested PCR analysis were used as previously described [1]. TaKaRa Taq DNA polymerase (TaKaRa Bio Inc., Tokyo, Japan) was used in all the PCR amplifications. A negative control with no DNA added was included in all PCR tests. All the secondary PCR products were subjected to electrophoresis in a 1.5\% agarose gel and visualized under UV by staining the gel with ethidium bromide.

\section{Nucleotide sequencing and analysis}

All the secondary PCR products of the expected size were directly sequenced with primers used for the secondary PCR after being purified on an ABI PRISM 3730 XL DNA Analyzer by Sinogeno-max Biotechnology Co. Ltd. (Beijing, China), using the Big Dye Terminator v3.1 Cycle Sequencing Kit (Applied Biosystems, USA). Sequence accuracy was confirmed by two-directional sequencing and by sequencing at least two new PCR products for some DNA preparations, from which we obtained sequences with nucleotide substitutions, deletions or insertions. To 
Table 1. Prevalence and genotypes of Enterocytozoon bieneusi in red deer and roe deer.

\begin{tabular}{|c|c|c|c|c|}
\hline Deer species (Latin name) & Location & Province & $\begin{array}{l}\text { No. positive/No. } \\
\text { examined }(\%)\end{array}$ & Genotype (n) \\
\hline \multirow[t]{2}{*}{ Red deer (Cervus elaphus) } & Great Hinggan Mountains & Heilongjiang & $3 / 44(6.8)$ & BEB6 (2); HLJD-VI (1) \\
\hline & Liaoyang & Liaoning & $5 / 60(8.3)$ & BEB6 (5) \\
\hline $\begin{array}{l}\text { Siberian roe deer } \\
\text { (Capreolus pygargus) }\end{array}$ & Liaoyang & Liaoning & $2 / 18(11.1)$ & BEB6 (2) \\
\hline Total & & & $10 / 122(8.2)$ & BEB6 (9); HLJD-VI (1) \\
\hline
\end{tabular}

Table 2. Prevalence and genotype distribution of Enterocytozoon bieneusi in red deer and roe deer by age and by gender.

\begin{tabular}{|c|c|c|c|c|c|c|c|}
\hline \multirow[t]{2}{*}{ Group } & & \multicolumn{3}{|c|}{ Red deer (Cervus elaphus) } & \multicolumn{3}{|c|}{ Siberian roe deer (Capreolus pygargus) } \\
\hline & & No. examined & No. positive (\%) & Genotype (n) & No. examined & No. positive (\%) & Genotype (n) \\
\hline \multirow[t]{2}{*}{ Age } & Young & 20 & $1(5.0)$ & BEB6 (1) & - & - & - \\
\hline & Adult & 84 & $7(8.3)$ & BEB6 (6); HLJD-VI (1) & 18 & $2(11.1)$ & BEB6 (2) \\
\hline \multirow[t]{2}{*}{ Gender } & Female & 74 & $6(8.1)$ & BEB6 (6) & 16 & $2(12.5)$ & BEB6 (2) \\
\hline & Male & 30 & $2(6.7)$ & BEB6 (1); HLJD-VI (1) & 2 & $0(0)$ & - \\
\hline
\end{tabular}

determine E. bieneusi genotypes, nucleotide sequences obtained in the present study were aligned with each other and reference sequences downloaded from the GenBank database using the Basic Local Alignment Search Tool (BLAST) and ClustalX1.83 (http://www.clustal.org/). If the sequences had $100 \%$ similarity with those from known genotypes, they would be given the first published name. If not, they were considered as novel genotypes. All the genotypes were named based on the $243 \mathrm{bp}$ of the ITS gene region of E.bieneusi, according to the established nomenclature system [24].

\section{Phylogenetic analysis}

To better present the diversity of all the genotypes obtained in the present study and the genetic relationship of novel ones to known ones, a comparison of the ITS region of all the nucleotide sequences obtained here and reference sequences previously published in GenBank was performed by implementing all the sequences obtained in Mega 5 software (http://www.megasoftware.net/). A neighbor-joining tree was generated based on the evolutionary distances calculated by the Kimura2-parameter model. The genogroup of the novel ITS genotype of E. bieneusi was designated. The confidence of the tree was assessed by bootstrap analysis using 1,000 replicates.

\section{Results and Discussion}

The present study was conducted to determine whether E. bieneusi was present in 122 deer fecal specimens by amplifying the ITS region of the rRNA gene. Overall, ten of the samples were PCR-positive and confirmed to be infected with E. bieneusi by sequence analysis, with an average infection rate of $8.2 \%(10 / 122)$. The infection rates by E. bieneusi were $11.1 \%(2 / 18)$ in red deer and $7.7 \%(8 / 104)$ in Siberian roe deer (Table 1). For red deer, $5.0 \%(1 / 20)$ were positive for E. bieneusi in the young animals and $8.3 \%(7 / 84)$ in the adults, while $8.1 \%$ $(6 / 74)$ were positive in females and $6.7 \%(2 / 30)$ in males (Table 2). None of the differences in infection rates were statistically significant either by gender $(\chi 2=0.03$, $p>0.05)$ or by age $\left(\chi^{2}=0.002, p>0.05\right)$. For Siberian roe deer, E. bieneusi was only found in two female adults (Table 2). This is the first report of E. bieneusi infection in Siberian roe deer. Molecular epidemiological data indicate that E. bieneusi has been found in all the deer species examined, including in the present study. To date, 137 deer-derived E. bieneusi isolates have been identified out of 852 deer. The infection rates by E. bieneusi varied from $7.1 \%$ to $75.0 \%$ in different deer species from different countries and areas (Table 3), with $75.0 \%$ in hog deer [17], $34.1 \%$ in Père David's deer [34], $16.8 \%$ in reindeer [19], $7.7-25.0 \%$ in red deer $[17,37$, this study], $7.1-50.0 \%$ in sika deer [17,33,37], 11.1\% in Siberian roe deer (this study), and $12.2-32.5 \%$ in white-tail deer $[10,26]$.

In the present study, two genotypes were identified in red deer and Siberian roe deer by sequencing and multiple sequence alignment, including one known genotype (BEB6) and a novel one (HLJD-VI; KY681138), which had only one base difference $(\mathrm{G} \rightarrow \mathrm{A}$ at the 207 nucleotide site) with genotype Peru6 (JF927955). A total of $90.0 \%$ (9/10) of E. bieneusi isolates were identified as genotype BEB6. In contrast, genotype HLJD-VI was only found in a male adult red deer. Genotype BEB6 (SH5) was originally detected in cattle in the USA [5]. This genotype has also been found in a sample from a child, as well as in water samples in China [29,31]. To date, this genotype has been found in at least 15 animal species from five countries, and the majority of BEB6 isolates are identified from herbivore animals in China, including deer, sheep, goats, cattle and horses (Table 4). These findings suggest that this genotype has a broad host range and geographical distribution as well as a zoonotic nature. Meanwhile, these observations also support the theory of host-parasite co-evolution in E. bieneusi. Related hosts have the same E.bieneusi 
Table 3. Prevalence and genotype distribution of Enterocytozoon bieneusi in natural infection of deer species worldwide.

\begin{tabular}{|c|c|c|c|c|}
\hline Deer species & $\begin{array}{l}\text { No. positive / } \\
\text { No. examined }(\%)\end{array}$ & $\begin{array}{l}\text { Genotype (n) } \\
\text { Group } 1\end{array}$ & Other Groups $^{\text {b }}$ & Reference \\
\hline Hog deer (Axis porcinus) & $3 / 4(75.0)$ & - & BEB6 (2); CHS9(1) & {$[17]$} \\
\hline $\begin{array}{l}\text { Père David's deer } \\
\text { (Elaphurus davidianus) }\end{array}$ & $16 / 47(34.0)$ & Type IV (4); EbpC (4); EbpA (4) & $\begin{array}{l}\text { BEB6 (2); COS-I (1); COS-II } \\
(1)\end{array}$ & [34] \\
\hline \multirow[t]{2}{*}{$\begin{array}{l}\text { Reindeer (Rangifer } \\
\text { tarandus) }\end{array}$} & $21 / 125(16.8)$ & $\begin{array}{l}\text { CHN-RD1(12); CHN-RD2(1); } \\
\text { CHN-RD3(1); CHN-RD4(1); } \\
\text { Peru6 (6) }\end{array}$ & - & [19] \\
\hline & $1 / 5(20.0)$ & - & HLJD-V (1) & {$[37]$} \\
\hline \multirow[t]{2}{*}{ Red deer (Cervus elaphus) } & $1 / 4(25.0)$ & - & BEB6 (1) & {$[17]$} \\
\hline & $8 / 104(7.7)$ & HLJD-VI (1) & $\operatorname{BEB} 6(7)$ & This study \\
\hline \multirow[t]{3}{*}{ Sika deer (Cervus nippon) } & $28 / 86(32.6)$ & $\begin{array}{l}\text { HLJD-II (1); HLJD-III (1); } \\
\text { HLJ-IV (1) }\end{array}$ & $\begin{array}{l}\text { BEB6 (20); HLJD-I (1); } \\
\text { HLJD-V (4) }\end{array}$ & {$[37]$} \\
\hline & $2 / 4(50.0)$ & SCO3 $(1)$ & $\operatorname{BEB} 6(1)$ & {$[17]$} \\
\hline & $23 / 326(7.1)$ & $\begin{array}{l}\text { CHN-DC-1 (1); KIN-1 (1); } \\
\text { EbpC (1); JLD-2 (2); JLD-3 (1) }\end{array}$ & J (11); BEB6 (4); JLD-1 (2) & {$[33]$} \\
\hline $\begin{array}{l}\text { Siberian roe deer } \\
\text { (Capreolus pygargus) }\end{array}$ & $2 / 18(11.1)$ & - & BEB6 (2) & This study \\
\hline & $6 / 49(12.2)$ & WL18 (2); WL19 (2) & WL4 (2) & {$[10]$} \\
\hline $\begin{array}{l}\text { White-tail deer } \\
\text { (Odocoileus leucurus) }\end{array}$ & $26^{\mathrm{a}} / 80(32.5)$ & LW1 (1) & $\begin{array}{l}\text { WL4 (11); I ( } 7) ; \mathrm{J}(1) \text {; } \\
\text { DeerEb1-DeerEb13 (one each) }\end{array}$ & {$[26]$} \\
\hline Total & $137 / 852(16.1)$ & $\begin{array}{l}\text { HLJD-II (1); HLJD-III (1); } \\
\text { HLJ-IV (2); Type IV (4); EbpC } \\
\text { (5); EbpA (4); CHN-RD1(12); } \\
\text { CHN-RD2(1); CHN-RD3(1); } \\
\text { CHN-RD4(1); Peru6 (6); } \\
\text { CHN-DC-1 (1); KIN-1 (1); JLD-2 } \\
\text { (2); JLD-3 (1); WL18 (2); WL19 } \\
\text { (2); LW1 (1); SCO3 (1) }\end{array}$ & $\begin{array}{l}\text { CHS9(1); BEB6 (39);HLJD-V } \\
\text { (5); HLJD-I (1); COS-I (1); } \\
\text { COS-II (1); J (12); JLD-1 (2); } \\
\text { WL4 (13); I (7); DeerEb1- } \\
\text { DeerEb13 (one each) }\end{array}$ & \\
\hline
\end{tabular}

${ }^{\mathrm{a}}$ : The numbers of genotypes are not consistent with the numbers of positives in White-tail deer due to mixed genotype infections [26].

${ }^{\mathrm{b}}$ : All the genotypes in the fourth column are in Group 2 except WL4 belonging to Group 3.

Table 4. Host ranges and geographical distribution of Enterocytozoon bieneusi genotype BEB6.

\begin{tabular}{|c|c|c|c|}
\hline Host & & Isolate no. $^{\mathrm{a}}$ & Reference \\
\hline \multicolumn{4}{|l|}{ Mammal } \\
\hline Human & Child & 1 & {$[29]$} \\
\hline Carnivora & Cat (Felis) & 2 & {$[13]$} \\
\hline \multirow[t]{11}{*}{ Ruminant } & Alpaca (Vicugna pacos) & 1 & {$[17]$} \\
\hline & Cattle (Bos taurus) & $2^{\mathrm{b}}$ & {$[5]$} \\
\hline & Golden takins (Budorcas taxicolor bedfordi) & 10 & {$[36]$} \\
\hline & Goat (Capra hircus) & $126^{\mathrm{c}}$ & {$[7,11,21,27,38]$} \\
\hline & Horse (Equus ferus caballus) & 9 & {$[23]$} \\
\hline & Hog deer (Axis porcinus) & 1 & {$[17]$} \\
\hline & Père David's deer (Elaphurus davidianus) & 16 & {$[34]$} \\
\hline & Red deer (Cervus elaphus) & 8 & {$[17,37$, This study $]$} \\
\hline & Sheep (Ovis aries) & $384^{\mathrm{d}}$ & {$[8,18,27,28,32,38]$} \\
\hline & Sika deer (Cervus nippon) & 27 & {$[17,33,37]$} \\
\hline & Siberian roe deer (Capreolus pygargus) & 2 & This study \\
\hline Rodent & Chinchillas (Chinchilla lanigera) & 3 & {$[22]$} \\
\hline Nonhuman primate & Nonhuman primate & 12 & {$[4,12,14]$} \\
\hline \multicolumn{4}{|l|}{ Bird } \\
\hline & Duck (Anas platyrhynchos domesticus) & 1 & {$[35]$} \\
\hline & Goose (Anser domestica) & 4 & {$[35]$} \\
\hline
\end{tabular}

${ }^{\mathrm{a}}$ : The isolates without annotations are only from China.

b : The two isolates are from the USA.

${ }^{\text {c }}$ : 125 isolates are from China with one from Peru.

d : 341 isolates are from China, with 32 and 11 from Sweden and Brazil, respectively. 
genotypes. Genotypes I and J are mainly found in cattle, sheep and goats $[38,39]$.

A high degree of genetic diversity has been observed within E. bieneusi in the ITS region of the rRNA gene. To date, at least 240 genotypes have been identified, including 40 genotypes identified in seven deer species (Table 3 ). Currently, it has been observed that 12 E. bieneusi genotypes identified in deer have been found in humans [20]. Meanwhile, most of the remaining genotypes from deer $(12 / 28,42.9 \%)$, including the novel genotype HLJDVI, are clustered into group 1 by phylogenetic analysis (data not shown). Group 1 contains $94 \%$ of the published ITS sequences of E. bieneusi, and almost all the humanpathogenic genotypes are in this group [20]. Thus, although some genotypes of E. bieneusi from deer have not been found in humans, the risk of their zoonotic transmission should not be neglected.

\section{Conclusion}

The present study reports the occurrence of E. bieneusi infection in Siberian roe deer for the first time. Based on the possibility of zoonotic transmission of deer-derived E. bieneusi isolates, and the fact that an increasing number of deer are bred in captivity, advice should be provide to those in contact with these animals, including farmers, breeders, veterinarians and animal handlers, to reduce the risk of zoonotic transmission of E. bieneusi.

\section{Competing interests}

The authors declare that they have no competing interests.

Acknowledgements. This study was supported by the Heilongjiang Province Education Bureau under grant No.12531266. The funders had no role in study design, data collection and analysis, the decision to publish, or preparation of the manuscript.

\section{References}

1. Buckholt MA, Lee JH, Tzipori S. 2002. Prevalence of Enterocytozoon bieneusi in swine: an 18-month survey at a slaughterhouse in Massachusetts. Applied and Environmental Microbiology, 68(5), 2595-2599.

2. Decraene V, Lebbad M, Botero-Kleiven S, Gustavsson AM, Löfdahl M. 2012. First reported food borne outbreak associated with microsporidia, Sweden, October 2009. Epidemiology and Infection, 140(3), 519-527.

3. Didier ES, Weiss LM. 2011. Microsporidiosis: not just in AIDS patients. Current Opinion in Infectious Diseases, 24(5), 490-495.

4. Du SZ, Zhao GH, Shao JF, Fang YQ, Tian GR, Zhang LX, Wang RJ, Wang HY, Qi M, Yu SK. 2015. Cryptosporidium spp., Giardia intestinalis, and Enterocytozoon bieneusi in captive non-human primates in Qinling Mountains. Korean Journal of Parasitology, 53(4), 395-402.

5. Fayer R, Santín M, Trout JM. 2007. Enterocytozoon bieneusi in mature dairy cattle on farms in the eastern United States. Parasitology Research, 102(1), 15-20.
6. Fayer R, Santin-Duran M. 2014. Epidemiology of Microsporidia in human infections, in Microsporidia: Pathogens of Opportunity, First Edition (Eds. L.M. Weiss and J. J. Becnel), John Wiley \& Sons, Inc., Chichester, UK. DOI: $10.1002 / 9781118395264 . c h 3$.

7. Feng Y, Li N, Dearen T, Lobo ML, Matos O, Cama V, Xiao. 2011. Development of a multilocus sequence typing tool for high-resolution genotyping of Enterocytozoon bieneusi. Applied and Environmental Microbiology, 77, 4822-4828.

8. Fiuza VR, Lopes CW, Cosendey RI, de Oliveira FC, Fayer R, Santín M. 2016. Zoonotic Enterocytozoon bieneusi genotypes found in brazilian sheep. Research in Veterinary Science, 107, 196-201.

9. Ghosh K, Weiss LM. 2009. Molecular diagnostic tests for microsporidia. Interdisciplinary perspectives on infectious diseases, 2009, 926521.

10. Guo Y, Alderisio KA, Yang W, Cama V, Feng Y, Xiao L. 2014. Host specificity and source of Enterocytozoon bieneusi genotypes in a drinking source watershed. Applied and Environmental Microbiology, 80(1), 218-225.

11. Jiang Y, Tao W, Wan Q, Li Q, Yang Y, Lin Y, Zhang S, Li W. 2015. Zoonotic and potentially host-adapted Enterocytozoon bieneusi genotypes in sheep and cattle in Northeast China and an increasing concern about the zoonotic importance of previously considered ruminant-adapted genotypes. Applied and Environmental Microbiology, 81, 3326-3335.

12. Karim MR, Dong H, Li T, Yu F, Li D, Zhang L, Li J, Wang R, Li S, Li X, Rume FI, Ning C. 2015. Predomination and new genotypes of Enterocytozoon bieneusi in captive nonhuman primates in zoos in China: high genetic diversity and zoonotic significance. PLoS One, 10(2), e0117991.

13. Karim MR, Dong H, Yu F, Jian F, Zhang L, Wang R, Zhang S, Rume FI, Ning C, Xiao L. 2014. Genetic diversity in Enterocytozoon bieneusi isolates from dogs and cats in China: host specificity and public health implications. Journal of Clinical Microbiology, 52(9), 3297-3302.

14. Karim MR, Wang R, Dong H, Zhang L, Li J, Zhang S, Rume FI, Qi M, Jian F, Sun M, Yang G, Zou F, Ning C, Xiao L. 2014. Genetic polymorphism and zoonotic potential of Enterocytozoon bieneusi from nonhuman primates in China. Applied and Environmental Microbiology, 80(6), 1893-1898.

15. Karim MR, Yu F, Li J, Li J, Zhang L, Wang R, Rume FI, Jian F, Zhang S, Ning C. 2014. First molecular characterization of enteric protozoa and the human pathogenic microsporidian, Enterocytozoon bieneusi, in captive snakes in China. Parasitology Research, 113(8), 3041-3048.

16. Li N, Xiao L, Wang L, Zhao S, Zhao X, Duan L, Guo M, Liu L, Feng Y. 2012. Molecular surveillance of Cryptosporidium spp., Giardia duodenalis, and Enterocytozoon bieneusi by genotyping and subtyping parasites in wastewater. PLoS Neglected Tropical Diseases, 6(9), e1809.

17. Li W, Deng L, Yu X, Zhong Z, Wang Q, Liu X, Niu L, Xie N, Deng J, Lei S, Wang L, Gong C, Zhou Z, Hu Y, Fu H, Xu H, Geng Y, Peng G. 2016. Multilocus genotypes and broad host range of Enterocytozoon bieneusi in captive wildlife at zoological gardens in China. Parasites \& Vectors, 9(1), 395.

18. Li W, Li Y, Li W, Yang J, Song M, Diao R, Jia H, Lu Y, Zheng J, Zhang X, Xiao L. 2014. Genotypes of Enterocytozoon bieneusi in livestock in China: high prevalence and zoonotic potential. PLoS One, 9, e97623.

19. Liu W, Nie C, Zhang L, Wang R, Liu A, Zhao W, Li H. 2015. First detection and genotyping of Enterocytozoon bieneusi in reindeers (Rangifer tarandus): a zoonotic potential of ITS genotypes. Parasites \& Vectors, 8, 526.

20. Matos O, Lobo ML, Xiao L. 2012. Epidemiology of Enterocytozoon bieneusi infection in Humans. Journal of Parasitology Research, 2012, 981424. 
21. Peng XQ, Tian GR, Ren GJ, Yu ZQ, Lok JB, Zhang LX, Wang XT, Song JK, Zhao GH. 2016. Infection rate of Giardia duodenalis, Cryptosporidium spp. and Enterocytozoon bieneusi in cashmere, dairy and meat goats in China. Infection, Genetics and Evolution, 41, 26-31.

22. Qi M, Luo N, Wang H, Yu F, Wang R, Huang J, Zhang L. 2015. Zoonotic Cryptosporidium spp. and Enterocytozoon bieneusi in pet chinchillas (Chinchilla lanigera) in China. Parasitology International, 64(5), 339-341.

23. Qi M, Wang R, Wang H, Jian F, Li J, Zhao J, Dong H, Zhu H, Ning C, Zhang L. 2016. Enterocytozoon bieneusi genotypes in grazing horses in china and their zoonotic transmission potential. Journal of Eukaryotic Microbiology, 63, 591-597.

24. Santín M, Fayer R. 2009. Enterocytozoon bieneusi genotype nomenclature based on the internal transcribed spacer sequence: a consensus. Journal of Eukaryotic Microbiology, $56(1), 34-38$.

25. Santín M, Fayer R. 2011. Microsporidiosis: Enterocytozoon bieneusi in domesticated and wild animals. Research in Veterinary Science, 90(3), 363-371.

26. Santin M, Fayer R. 2015. Enterocytozoon bieneusi, Giardia, and Cryptosporidium infecting white-tailed deer. Journal of Eukaryotic Microbiology, 62, 34-43.

27. Shi K, Li M, Wang X, Li J, Karim MR, Wang R, Zhang L, Jian F, Ning C. 2016. Molecular survey of Enterocytozoon bieneusi in sheep and goats in China. Parasites \& Vectors, $9,23$.

28. Stensvold CR, Beser J, Ljungström B, Troell K, Lebbad M. 2014. Low host-specific Enterocytozoon bieneusi genotype BEB6 is common in Swedish lambs. Veterinary Parasitology, 205(1-2), 371-374.

29. Wang L, Xiao L, Duan L, Ye J, Guo Y, Guo M, Liu L, Feng Y. 2013. Concurrent infections of Giardia duodenalis, Enterocytozoon bieneusi, and Clostridium difficile in children during a cryptosporidiosis outbreak in a pediatric hospital in China. PLoS Neglected Tropical Diseases, 7(9), e2437.

30. Yang Z, Zhao W, Shen Y, Zhang W, Shi Y, Ren G, Yang D, Ling H, Yang F, Liu A, Cao J. 2016. Subtyping of Cryptosporidium cuniculus and genotyping of Enterocyto- zoon bieneusi in rabbits in two farms in Heilongjiang Province, China. Parasite, 23, 52.

31. Ye J, Ji Y, Xu J, Ma K, Yang X. 2017. Zoonotic Enterocytozoon bieneusi in raw waste water in Zhengzhou, China. Folia Parasitologica (Praha), 64, 002.

32. Ye J, Xiao L, Wang Y, Guo Y, Roellig DM, Feng Y. 2015. Dominance of Giardia duodenalis assemblage A and Enterocytozoon bieneusi genotype BEB6 in sheep in Inner Mongolia, China. Veterinary Parasitology, 210(3-4), 235-239.

33. Zhang XX, Cong W, Liu GH, Ni XT, Ma JG, Zheng WB, Zhao Q, Zhu XQ. 2016. Prevalence and genotypes of Enterocytozoon bieneusi in Sika deer in Jilin province, Northeastern China. Acta Parasitologica, 61(2), 382-388.

34. Zhang Z, Huang J, Karim MR, Zhao J, Dong H, Ai W, Li F, Zhang L, Wang R. 2015. Zoonotic Enterocytozoon bieneusi genotypes in Père David's deer (Elaphurus davidianus) in Henan, China. Experimental Parasitology, 155, 46-48.

35. Zhao W, Yu S, Yang Z, Zhang Y, Zhang L, Wang R, Zhang W, Yang F, Liu A. 2016. Genotyping of Enterocytozoon bieneusi (Microsporidia) isolated from various birds in China. Infection, Genetics and Evolution, 40, 151-154.

36. Zhao GH, Du SZ, Wang HB, Hu XF, Deng MJ, Yu SK, Zhang LX, Zhu XQ. 2015. First report of zoonotic Cryptosporidium spp., Giardia intestinalis and Enterocytozoon bieneusi in golden takins (Budorcas taxicolor bedfordi). Infection, Genetics and Evolution, 34, 394-401.

37. Zhao W, Zhang W, Wang R, Liu W, Liu A, Yang D, Yang F, Karim MR, Zhang L. 2014. Enterocytozoon bieneusi in sika deer (Cervus nippon) and red deer (Cervuselaphus): deer specificity and zoonotic potential of ITS genotypes. Parasitology Research, 13(11), 4243-4250.

38. Zhao W, Zhang W, Yang D, Zhang L, Wang R, Liu A. 2015. Prevalence of Enterocytozoon bieneusi and genetic diversity of ITS genotypes in sheep and goats in China. Infection, Genetics and Evolution, 32, 265-270.

39. Zhao W, Zhang W, Yang F, Zhang L, Wang R, Cao J, Shen Y, Liu A. 2015. Enterocytozoon bieneusi in dairy cattle in the Northeast of China: Genetic diversity of ITS gene and evaluation of zoonotic transmission potential. Journal of Eukaryotic Microbiology, 62(4), 553-560.

Cite this article as: Zhao W, Wang J, Yang Z, Liu A. 2017. Dominance of the Enterocytozoon bieneusi genotype BEB6 in red deer (Cervus elaphus) and Siberian roe deer (Capreolus pygargus) in China and a brief literature review. Parasite 24, 54

\section{PARASTIE}

An international open-access, peer-reviewed, online journal publishing high quality papers on all aspects of human and animal parasitology

Reviews, articles and short notes may be submitted. Fields include, but are not limited to: general, medical and veterinary parasitology; morphology, including ultrastructure; parasite systematics, including entomology, acarology, helminthology and protistology, and molecular analyses; molecular biology and biochemistry; immunology of parasitic diseases; host-parasite relationships; ecology and life history of parasites; epidemiology; therapeutics; new diagnostic tools.

All papers in Parasite are published in English. Manuscripts should have a broad interest and must not have been published or submitted elsewhere. No limit is imposed on the length of manuscripts.

Parasite (open-access) continues Parasite (print and online editions, 1994-2012) and Annales de Parasitologie Humaine et Comparée (1923-1993) and is the official journal of the Société Française de Parasitologie. 